\title{
Observation of Transition Metal Dissolution at Cathode Surface
}

Lei Yu${ }^{1}$, Tongchao Liu ${ }^{1}$, Junwu Zhu ${ }^{2}$, Jun $\mathrm{Lu}^{1}$ and Jianguo Wen ${ }^{1}$

${ }^{1}$ Argonne National Laboratory, Lemont, Illinois, United States, ${ }^{2}$ Nanjing University of Science and Technology, Nanjing, Jiangsu, China (People's Republic)

Transition metal (TM) dissolution has been widely reported to severely degrade the anode, which is historically long accepted to be a singular root cause of battery capacity fading [1]. However, its impact on cathode behavior remains poorly understood. In spite of elaborate investigations into TM migration mechanisms on cathode surfaces, structural evolution of cathode materials undergoing TM dissolution and their interrelationships still have not been clear [2]. In this study, commercial lithium manganite $\left(\mathrm{LiMn}_{2} \mathrm{O}_{4}\right)$ is chosen as the investigated model material owing to its pronounced $\mathrm{Mn}$ (II) ion dissolution. By using aberration-corrected high-resolution TEM (HRTEM) and electron energy loss spectroscopy (EELS), it is found that many microcracks caused by structure phase transition and strain are generated on the cycled cathode particle surfaces. At the crack area, although there is no obvious lattice distinction, the electronic structure shows remarkable changes. The presence of $\mathrm{Mn}$ (II) indicates the TM dissolution prevails at the crack. The synergy effect of TM dissolution and irreversible structure damage may collectively accelerate cathode deterioration.

For TEM observation, a small piece of $\mathrm{LiMn}_{2} \mathrm{O}_{4}$ electrode after 25 cycles was ultrasonically dispersed in isopropanol, and then the obtained suspension was dropped on a copper grid. Low-magnification TEM was firstly exploited to investigate the morphology of cycled electrode material. Fig. 1 presents the resulting TEM images captured from different $\mathrm{LiMn}_{2} \mathrm{O}_{4}$ particle surfaces. It is clear that some split cracks appear on the particle surfaces after electrochemical cycles. Especially, it is also noticed some other premature cracks inside the particle (yellow marked areas), which effectively demonstrate that these cracks are resulted from the irreversible structure transformation and strain instead of mechanical damage.

The detailed structure characteristic at the crack was ascertained by HRTEM. Fig. $2 \mathbf{b}$ is corresponding HRTEM images of a crack in Fig. 2a. There is no obviously distinguishable difference in lattice parameters between crack and bulk. Hence, EELS was further probed their fine electronic structural evolution. In the Mn L-edge (Fig. 2d), at the crack, both of the peaks Mn-L3 and Mn-L2 exhibit chemical shifts towards lower energy loss and a higher intensity ratio of $\mathrm{L}_{3} / \mathrm{L}_{2}$, which implies the decrease of $\mathrm{Mn}$ valence [3]. In the oxygen K-edge (Fig. 2c), we can observe two main peaks labeled as a and b. At the crack, the intensity of peak a1 decreases and $a_{2}$ increases, and peak a shifts towards higher energy loss and peak $b$ shifts towards lower energy loss, which exactly matches with that of $\mathrm{Mn}_{3} \mathrm{O}_{4}$ reported in the earlier literature [4]. As a result, both edges show the decrease in Mn valence from bulk to crack, and the crack exhibits the mix Mn valence of $2^{+} / 3^{+}$, illustrating the occurrence of Mn disproportionation reaction $[5]$. 

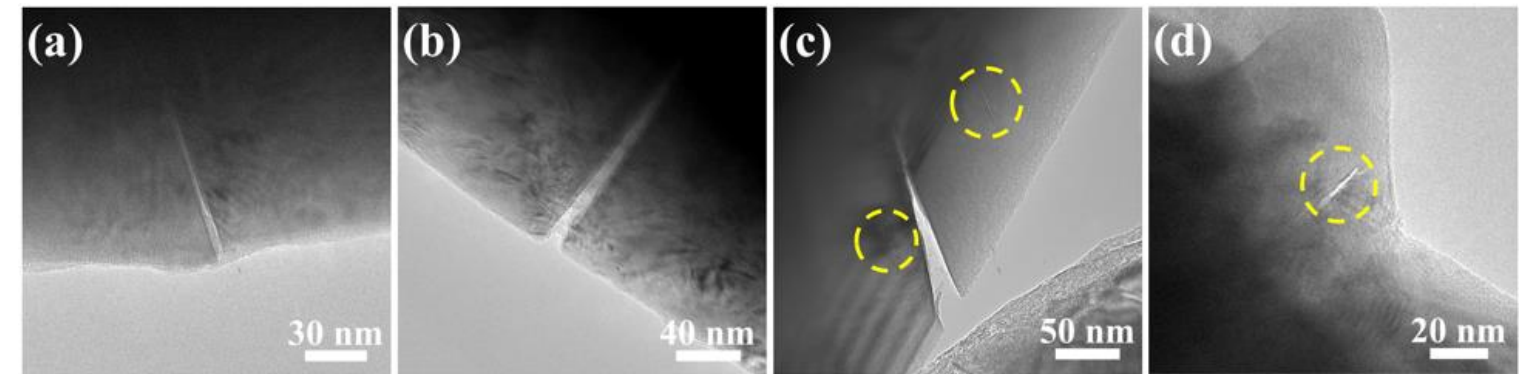

Figure 1. Low-magnification TEM images of cracks captured in different cycled LiMn2O4 particles.
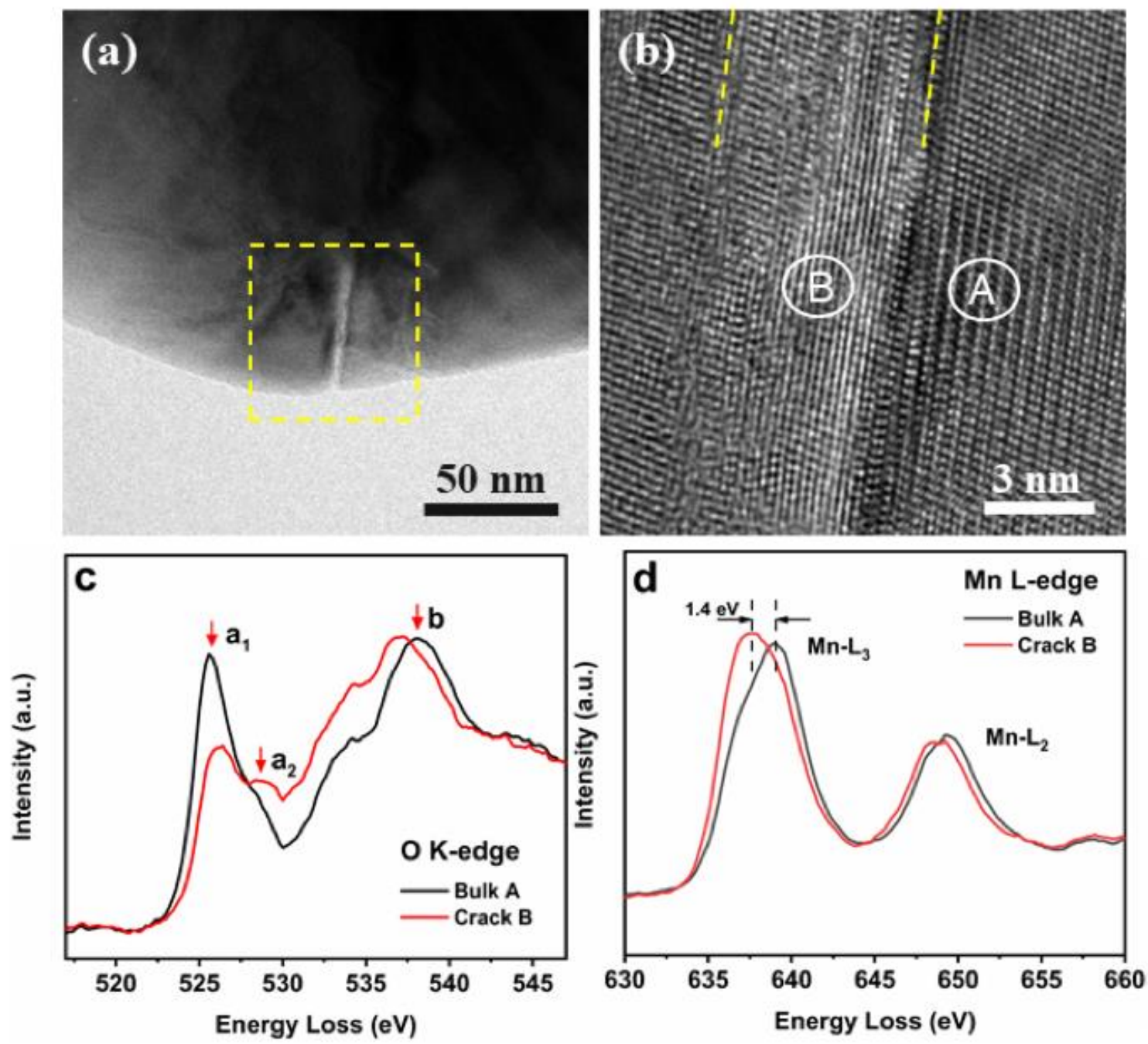

Figure 2. Decrease of Mn valence at crack compared with that in bulk. Low-magnification TEM (a) and corresponding HRTEM (b) images at a crack in LiMn2O4 after 25 cycles. Typical EELS spectra of O Kedge (c) and Mn L-edges (d) measured at bulk (A) and crack (B) as shown in (b).

\section{References}

[1] I. A. Shkrob, A. J. Kropf, T. W. Marin, Y. Li, O. G. Poluektov, J. Niklas and D. P. Abraham, J. Phys. Chem. C, 2014, 118, 24335-24348.

[2] K. Leung, Chem. Mater., 2017, 29, 2550-2562.

[3] G. Botton, C. Appel, A. Horsewell and W. Stobbs, J. Microsc., 1995, 180, 211-216. 
[4] D. Tang, Y. Sun, Z. Yang, L. Ben, L. Gu and X. Huang, Chem. Mater., 2014, 26, 3535-3543. [5] This work was performed at the Center for Nanoscale Materials, a U.S. Department of Energy Office of Science User Facility, and supported by the U.S. Department of Energy, Office of Science, under Contract No. DE-AC02-06CH11357. Lei $\mathrm{Yu}$ is grateful for the financial support from the China Scholarship Council (No 201806840106). 